Revisión de la literatura 


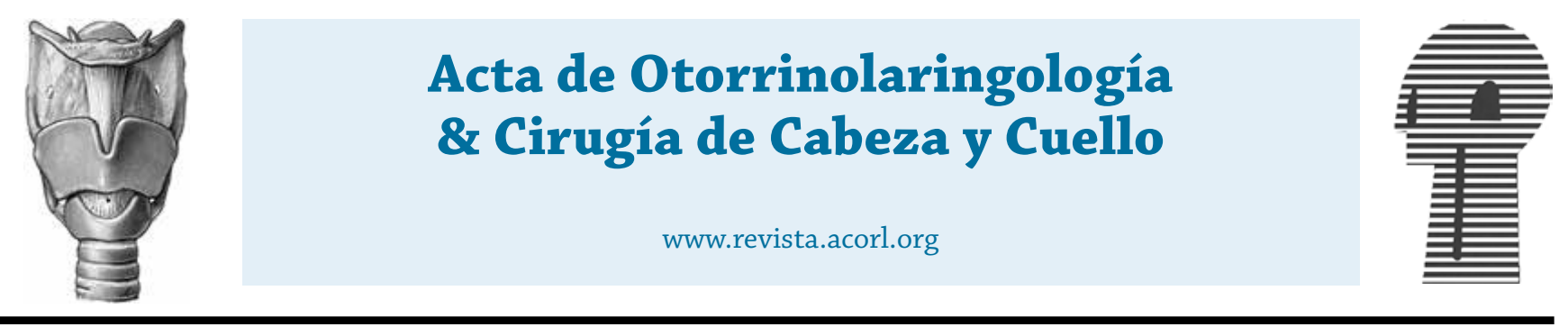

\title{
Ayudas diagnósticas en patología laríngea: imagen de banda estrecha $(\mathrm{NBI})$
}

\section{Diagnostic tools in laringeal patology: narrow band imaging (NBI)}

\author{
Zamora-Cely $L^{*}$, Gómez-Rodríguez $A E^{* *}$, Perea-Mena IL ${ }^{* * *}$, Moncada-Sánchez IF****
}

* Otorrinolaringóloga, Fundación Universitaria de Ciencias de la Salud - Hospital de San José de Bogotá.

** Otorrinolaringólogo, Instructor asociado, servicio de otorrinolaringología, Fundación Universitaria de Ciencias de la Salud - Hospital de San José de Bogotá.

*** Residente de cuarto año de otorrinolaringología, Fundación Universitaria de Ciencias de la Salud - Hospital de San José de Bogotá.

**** Residente de cuarto año de otorrinolaringología, Fundación Universitaria de Ciencias de la salud - Hospital de San José de Bogotá.

Forma de citar: Zamora-Cely LM, Gómez-Rodríguez AE, Perea-Mena IL, Moncada-Sánchez IF. Ayudas diagnósticas en patología laríngea: imagen de banda estrecha (NBI). Acta otorrinolaringol. cir. cabeza cuello. 2020; 48(3):240-248 Doi:10.37076/acorl.v48i3.521

\section{INFORMACIÓN DEL ARTÍCULO}

Historia del artículo:

Recibido: 20 de mayo de 2020

Evaluado: 19 de agosto de 2020

Aceptado: 1 de septiembre de 2020

Palabras clave (DeCS):

enfermedades de la laringe,

diagnóstico por imagen, neoplasias laríngeas.

\section{RESUMEN}

La imagen de banda estrecha (narrow band imaging, NBI) es un sistema de mejora de imagen desarrollado en Japón en 2001, que utiliza longitudes de onda filtradas a $415 \mathrm{~nm}$ y $540 \mathrm{~nm}$ que permiten la visualización de anomalías microvasculares asociadas a la carcinogénesis. En laringología, la NBI se usa para evaluar lesiones premalignas, detectar neoplasias primarias de origen desconocido en la cabeza y el cuello, diagnosticar y hacer un seguimiento de lesiones malignas después de la realización de un tratamiento endoscópico y/o con radioterapia, entre otros. En este artículo se presenta una revisión narrativa de la literatura, en la que se exponen los fundamentos técnicos de la NBI, los principios fisiológicos básicos, las clasificaciones actuales para describir los diferentes patrones vasculares y los usos principales

\section{Correspondencia:}

Dr. Álvaro Gómez Rodríguez

E-mail: alvarogomez10@hotmail.com

Dirección: Carrera 9 \# 126-15, apto 205

Teléfono celular: 3118209277 
descritos en laringología, así como un atlas de imágenes tomadas en el servicio de otorrinolaringología del Hospital de San José.

\section{ABSTRACT}

Key words (MeSH):

laryngeal diseases, diagnostic imaging, laryngeal neoplasms.
Narrow band imaging (NBI) is an image enhancement system developed in Japan in 2001, which uses wavelengths filtered at $415 \mathrm{~nm}$ and $540 \mathrm{~nm}$ that allow the visualization of microvascular anomalies, that have been associated with carcinogenesis. In laryngology, the NBI is used for the evaluation of premalignant lesions, detection of unknown primary neoplasm in head and neck, diagnosis and follow-up of malignant lesions after endoscopic treatment and/or with radiotherapy, among others. This narrative review of the literature is about technical foundations of the NBI, the basic physiological principles, the current classifications to describe the different vascular patterns and the main uses described in laryngology, as well as an atlas of images taken in the Otolaryngology service from the Hospital de San José.

\section{Introducción}

La imagen de banda estrecha (narrow band imaging, $N B I$ ) es un sistema de imagen desarrollado en Japón en 2001 y avalado por la Administración de Medicamentos y Alimentos (Food and Drug Administration, FDA) en 2009 (1, 2). Este usa longitudes de onda filtradas que mejoran la visualización de estructuras microvasculares mucosas, cuyas anomalías se asocian con lesiones preneoplásicas y neoplásicas, uno de los primeros cambios observados $(3,4)$. De esta manera, la NBI puede superar de manera efectiva la mayoría de las limitaciones descritas para las tinciones supravitales y la autofluorescencia, con una reducción en el número de falsos positivos $(3,5)$.

En 2004 empieza a estudiarse la precisión de la NBI en el diagnóstico del carcinoma de vía aerodigestiva superior. En 2010 se publicó el primer trabajo sobre la utilidad de la NBI en las neoplasias primarias de origen desconocido, el cual concluyó que tiene un valor complementario para la localización del tumor de origen (1). En 2012, Lin y colaboradores establecen que ciertos patrones de la NBI son útiles para el diagnóstico precoz del carcinoma epidermoide en el piso de la boca, la mucosa ventral de la lengua, el paladar blando, los pilares amigdalinos, las amígdalas palatinas, la base de lengua, la pared posterior de orofaringe e hipofaringe; es decir, zonas con el epitelio fino no queratinizado (6).

Sin embargo, existían dudas sobre la utilidad de los diferentes patrones para mejorar la exactitud del diagnóstico en las áreas cubiertas con epitelio no queratinizado grueso o epitelio queratinizado como la glotis (7). Posteriormente, $\mathrm{Ni}$ y colaboradores publicaron un estudio sobre la concordancia entre los patrones de neoangiogénesis y carcinoma, en el que establecieron una especificidad de $93,2 \%$, una sensibilidad de $88,9 \%$ y un valor predictivo positivo de $90 \%$ (8).

Actualmente, la utilidad de la NBI en la laringología es variada: para el diagnóstico preoperatorio, para la evaluación de los bordes libres de tumor durante la cirugía y en el seguimiento para la detección de recurrencias en las diferentes modalidades de tratamiento (7).
En Colombia no existen estudios acerca de la utilidad de la NBI y las ventajas que puede traer para los pacientes con una patología laríngea, dado que se encuentra en pocas instituciones en el país, entre ellas el servicio de otorrinolaringología del Hospital de San José de Bogotá. Allí se utiliza de manera rutinaria con fines pedagógicos, razón por la cual se decide realizar esta revisión de la literatura como punto de partida para futuros estudios.

\section{Fundamentos técnicos}

La NBI modifica el ancho de banda de la luz blanca mediante un sistema de dos filtros que seleccionan el espectro azul y el espectro verde, los cuales corresponden a los picos de absorción de la hemoglobina. El filtro de luz azul, con una longitud de onda centrada en $415 \mathrm{~nm}$, se refleja fundamentalmente en la superficie, realzando el lecho capilar que aparece como una imagen de color marrón. El filtro de luz verde, con una longitud de onda centrada en $540 \mathrm{~nm}$, penetra en profundidad y realza las imágenes del plexo vascular de la submucosa, tejido conectivo $\mathrm{y}$, por lo tanto, permite una mejor visualización de las arteriolas y las vénulas (7).

Además de los videoendoscopios transnasales de diferentes tamaños, las cámaras NBI también se pueden aplicar a lentes rígidos de diferentes ángulos para evaluar la laringe con anestesia local y general. En el servicio de otorrinolaringología del Hospital de San José utilizamos un videoendoscopio flexible HD y un videoprocesador VH 170 de Olympus HD (Figura 1).

La definición de imagen óptima tanto para la luz blanca convencional como para la endoscopia NBI se logra utilizando una cámara de televisión de alta definición (HDTV), que proporciona 1080 líneas de resolución, lo que permite una definición de señal 4,26 veces mejor que la endoscopia estándar.

En el servicio de otorrinolaringología del Hospital de San José, el examen inicia con la aplicación de anestesia tópica nasal (lidocaína al $2 \%$ sin epinefrina) en la base de lengua, la epiglotis y la cara superficial de ambas cuerdas con cánula de 


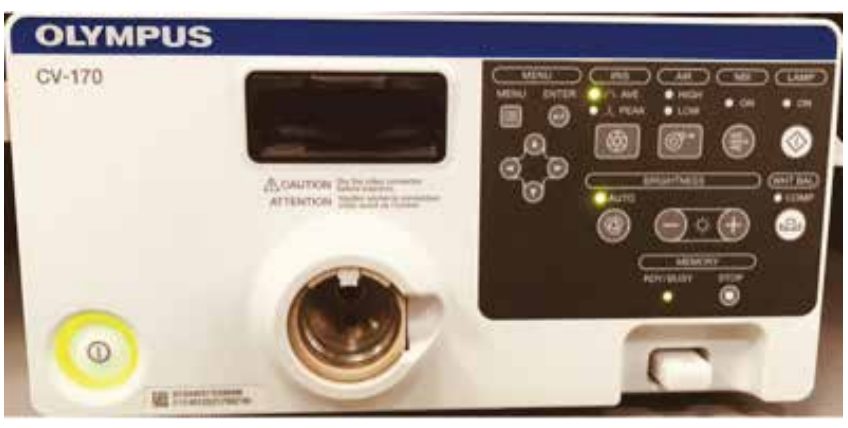

Figura 1. Videoprocesador Olympus CV-170. Imagen cortesía del servicio de otorrinolaringología del Hospital de San José.

Abraham; esto permite aproximar el endoscopio lo suficiente para identificar los diferentes patrones de neoangiogénesis, al abolir el reflejo nauseoso. Cabe destacar que es importante tener una buena intensidad de luz, ya que es un factor decisivo en la observación de cambios patológicos. Se pasa de la luz blanca al modo NBI y se hace zoom en las lesiones presionando un botón en el videoendoscopio, la videocámara o la consola del monitor (3) (Figuras 2 y 3 ).

\section{Principios básicos}

\section{Microcirculación de las cuerdas vocales}

La microcirculación de las cuerdas vocales se caracteriza por la presencia de capilares en la lámina propia superficial, y arterias y venas más pequeñas en las capas más profundas. En cuanto a la estructura vascular de la lámina propia, está bien separada de la capa muscular y solo unos pocos vasos surgen directamente del músculo vocal subyacente y alcanzan la lámina propia (10).
En condiciones fisiológicas, los vasos del lado ventricular del pliegue vocal tienen un curso uniforme, moderadamente ondulado y serpenteante con ramas dispersas, que se asemejan a un árbol. En el modo NBI, la mucosa laríngea normal consiste en los vasos submucosos (que aparecen de color verde), que se conectan con una red vascular arborescente (que aparece de color marrón oscuro) (10) (Figura 4).

\section{Clasificaciones}

Las anomalías de la vasculatura de los pliegues vocales generalmente se clasifican de acuerdo con sus cambios de forma respecto a los patrones normales. Se ha encontrado que tales cambios predicen la profundidad de la invasión de cáncer superficial y se clasifican en cinco categorías diferentes (Clasificación de Ni) (Tabla 1) (11, 12).

Las lesiones observadas en NBI se registran generalmente como $(13,14)$ :

- Malignas (tipo V).

- Con sospecha de malignidad (lesiones protuberantes o ulcerativas cubiertas con tejido necrótico o leucoplasia de tipo desconocido).

- Benignas (tipos I a IV).

Sin embargo, los autores consideran que a pesar de que la Clasificación de Ni se usa ampliamente, hay algunas fallas por resolver. En primer lugar, las lesiones de tipo IV no podrían considerarse únicamente benignas, sino también podrían representar epitelio/displasia de alto riesgo y malignidad; en segundo lugar, la estructura de la clasificación podría simplificarse (8). En 2015, la ELS propuso una nueva clasificación, la cual dividía los patrones vasculares en dos grupos (4):

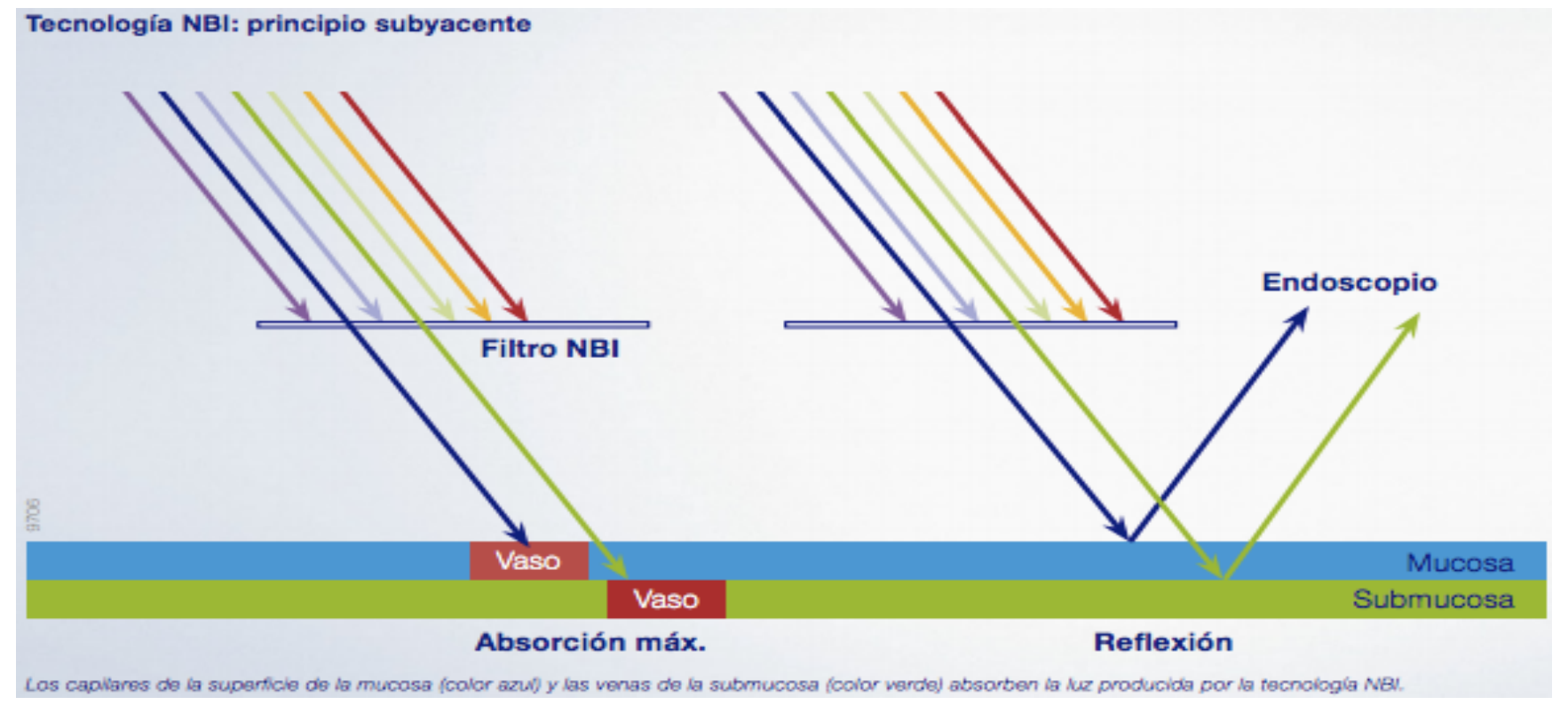

Figura 2. Principio de imagen de banda estrecha - absorción de luz por los capilares. Imagen tomada con la autorización del grupo Olympus Latinoamérica. Tomada de la referencia 9. 


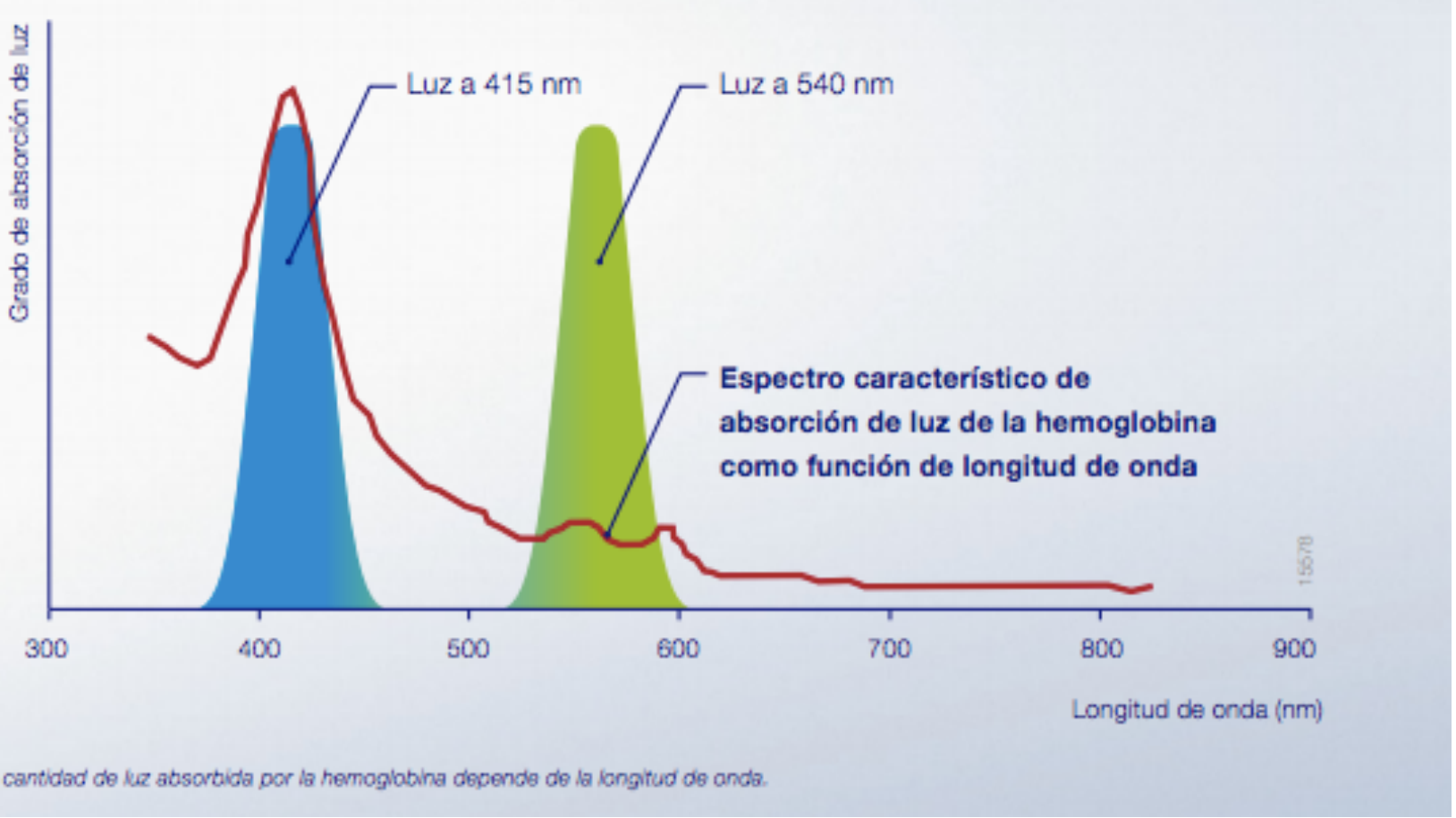

Figura 3. Principio de imagen de banda estrecha - espectro de absorción de luz respecto a la longitud de onda. Imagen tomada con autorización del grupo Olympus Latinoamérica. Tomada de la referencia 9.
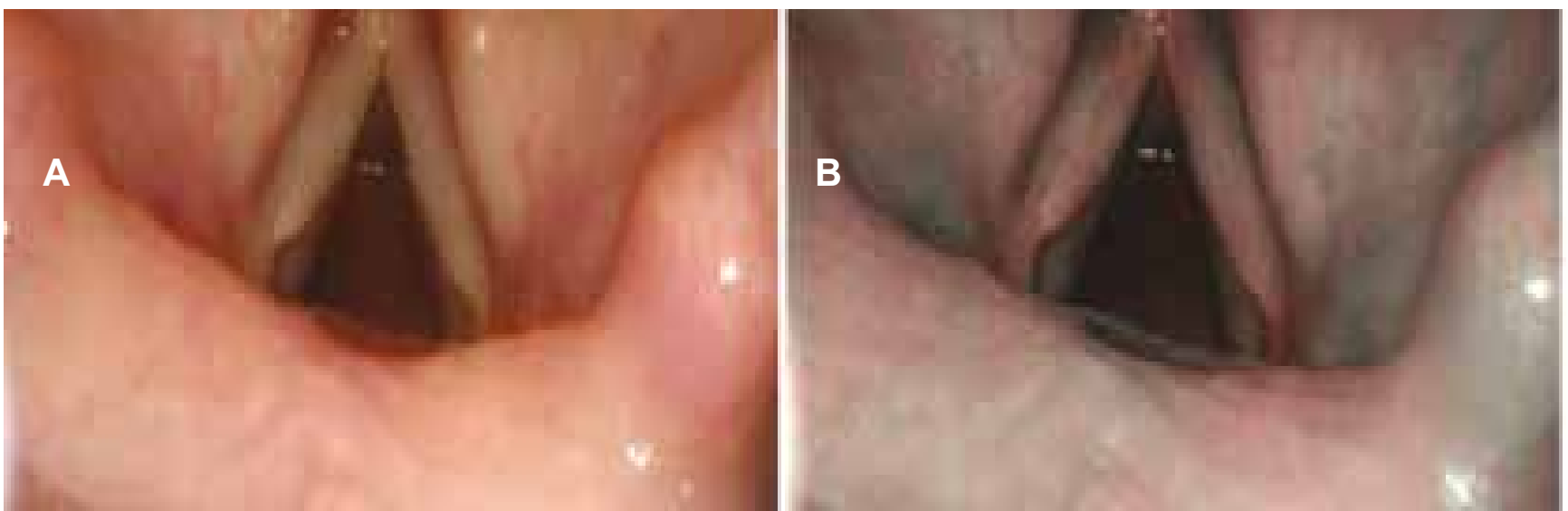

Figura 4. Patrón vascular de una laringe normal. A. Imagen con luz blanca. B. Imagen con NBI. Imágenes cortesía del servicio de otorrinolaringología del Hospital de San José.

- Cambios vasculares longitudinales

- Cambios vasculares perpendiculares

\section{Cambios vasculares longitudinales}

Los cambios patológicos de la vasculatura de los pliegues vocales empiezan con vasos sanguíneos dilatados, también conocidos como ectasia vascular. Posteriormente las paredes de los vasos se vuelven porosas, inestables y permeables, lo que produce un cambio de forma constante. Esto da como resultado otros vasos ectásicos, dilatados con paredes de vasos lobulados, que presentan la imagen típica del curso serpenteante (7).
Los procesos inflamatorios conducen a angiogénesis, que produce un aumento del número y la densidad de vasos, la cual se asocia con una mayor ramificación y anastomosis entre ellos (5).

Los vasos sanguíneos con cambios en la dirección transversal se conocen como indicador, índice o nutricio, dado que nutre y mantiene diversos cambios en los tejidos de las cuerdas vocales, como pólipos, quistes o carcinomas (Figura 5) (15).

\section{Cambios vasculares perpendiculares}

En contraste con los patrones longitudinales que se extienden solamente a lo largo y ancho de las cuerdas vocales, los 
Tabla 1. Clasificación de Ni y de la Sociedad Europea de Laringología (European Laringological Society, ELS)

\begin{tabular}{|c|c|c|c|c|}
\hline \multicolumn{3}{|c|}{ Clasificación de $\mathrm{Ni}$} & \multicolumn{2}{|c|}{ ELS } \\
\hline Patrón & Descripción & Imagen & \multirow{5}{*}{ 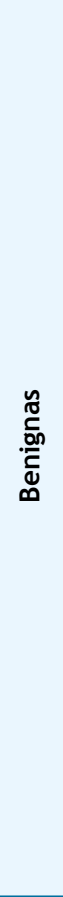 } & \multirow{5}{*}{ 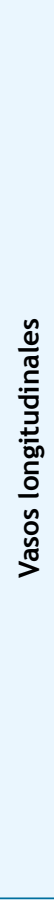 } \\
\hline Tipo 1: lesiones benignas & $\begin{array}{l}\text { Los bucles capilares } \\
\text { papilares intraepiteliales } \\
\text { son casi imperceptibles } \\
\text { pero se pueden ver } \\
\text { claramente vasos oblicuos y } \\
\text { arborescentes de pequeño } \\
\text { diámetro. }\end{array}$ & & & \\
\hline $\begin{array}{l}\text { Tipo 2: lesiones } \\
\text { benignas, laringitis } \\
\text { crónica }\end{array}$ & $\begin{array}{l}\text { capilares papilares } \\
\text { intraepiteliales también son } \\
\text { casi imperceptibles, pero } \\
\text { el diámetro de los vasos } \\
\text { oblicuos y arborescentes ] }\end{array}$ & & & \\
\hline $\begin{array}{l}\text { Tipo 3: lesiones } \\
\text { benignas, leucoplaquias, } \\
\text { displasia de bajo grado }\end{array}$ & $\begin{array}{l}\text { La visualización de los } \\
\text { capilares está parcialmente } \\
\text { obstruida por una capa de } \\
\text { hiperqueratosis (llamado } \\
\text { efecto sombrilla); no se } \\
\text { visualizan bucles capilares } \\
\text { papilares intraepiteliales }\end{array}$ & & & \\
\hline $\begin{array}{l}\text { Tipo 4: displasia de grado } \\
\text { medio y alto }\end{array}$ & $\begin{array}{l}\text { Los bucles capilares } \\
\text { papilares intraepiteliales se } \\
\text { reconocen como pequeños } \\
\text { puntos }\end{array}$ & & & \\
\hline $\begin{array}{l}\text { Tipo 5a: displasia de alto } \\
\text { grado, carcinoma in situ }\end{array}$ & $\begin{array}{l}\text { Los bucles capilares } \\
\text { papilares intraepiteliales } \\
\text { aparecen como sólidos } \\
\text { o huecos con un patrón } \\
\text { marrón o moteado de varias } \\
\text { formas. }\end{array}$ & & \multirow{3}{*}{ 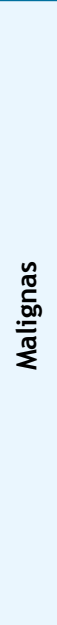 } & \multirow{3}{*}{ 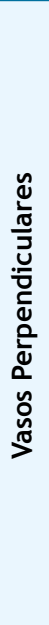 } \\
\hline $\begin{array}{l}\text { Tipo 5b: carcinoma in } \\
\text { situ, cáncer invasivo }\end{array}$ & $\begin{array}{l}\text { Los bucles capilares } \\
\text { papilares intraepiteliales } \\
\text { aparecen como líneas } \\
\text { irregulares tortuosas }\end{array}$ & & & \\
\hline $\begin{array}{l}\text { Tipo 5c: carcinoma in } \\
\text { situ, cáncer invasivo }\end{array}$ & $\begin{array}{l}\text { Los bucles capilares } \\
\text { papilares intraepiteliales } \\
\text { aparecen como motas } \\
\text { marrones o formas } \\
\text { tortuosas con una } \\
\text { distribución irregular sobre } \\
\text { la superficie del tumor }\end{array}$ & $\angle \infty$ & & \\
\hline
\end{tabular}

patrones perpendiculares se proyectan perpendicularmente hacia la superficie del epitelio. Se reconocen endoscópicamente como bucles capilares papilares intraepiteliales. El ángulo del punto de inflexión de los bucles puede ser ancho o estrecho, lo que corresponde al papiloma y la lesión maligna, respectivamente $(10,11,16)$ (Figura 6).

En este sentido, la clasificación propuesta por la ELS se puede usar de forma efectiva para diferenciar la enfermedad maligna de benigna, la cual se puede aplicar de forma más sencilla específicamente para otorrinolaringólogos con menos experiencia. De acuerdo con esta, la endoscopia con NBI debe realizarse en dos pasos: evaluación de un posible patrón vascular perpendicular para diferenciar entre una posible malignidad y una enfermedad benigna; en presencia de un patrón perpendicular, el paso dos definirá los puntos de inflexión de ángulo estrecho y ángulo amplio de los bucles capilares papilares intraepiteliales, que diferencia entre carcinoma y papiloma, respectivamente (4).

\section{Indicaciones}

\section{Lesiones premalignas}

Son lesiones secundarias a una inflamación prolongada de la mucosa laríngea causada por diferentes factores etiológicos, los cuales producen cambios macroscópicos e histológicos, incluida su posible transformación maligna (7).

\section{Leucoplasia, paquidermia o papiloma escamoso}

Son las más frecuentes (60\% de las laringitis crónicas inespecíficas), y más del $15 \%$ se correlacionan con displasias graves. En estas lesiones, la NBI permite detectar cambios 

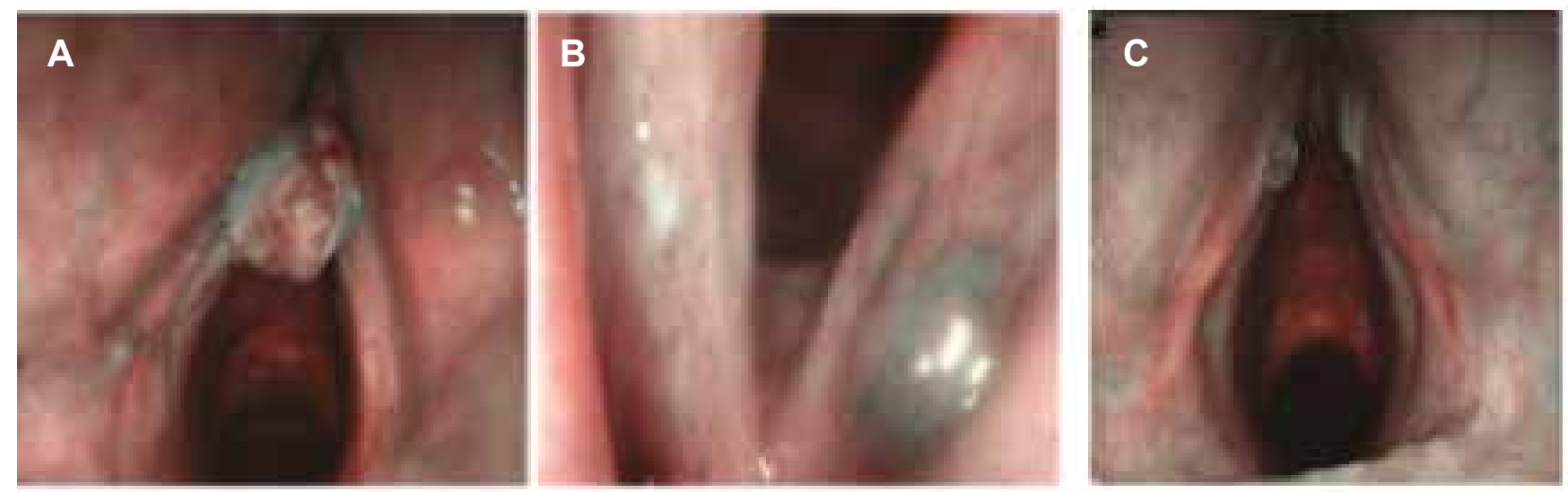

Figura 5. Vasos nutricios. A. Lesión exofítica del tercio medio y anterior en la cuerda vocal izquierda con vaso nutricio, que muestra un patrón longitudinal y serpenteante B. Lesión quística en el tercio medio de la cuerda vocal derecha con vasos nutricios longitudinales y arborescentes, con ramificaciones que rodean la lesión C. Lesión polipoidea en el tercio medio de la cuerda vocal izquierda, con una leucoplaquia contralateral y vaso nutricio longitudinal. Imágenes cortesía del servicio de otorrinolaringología del Hospital de San José.
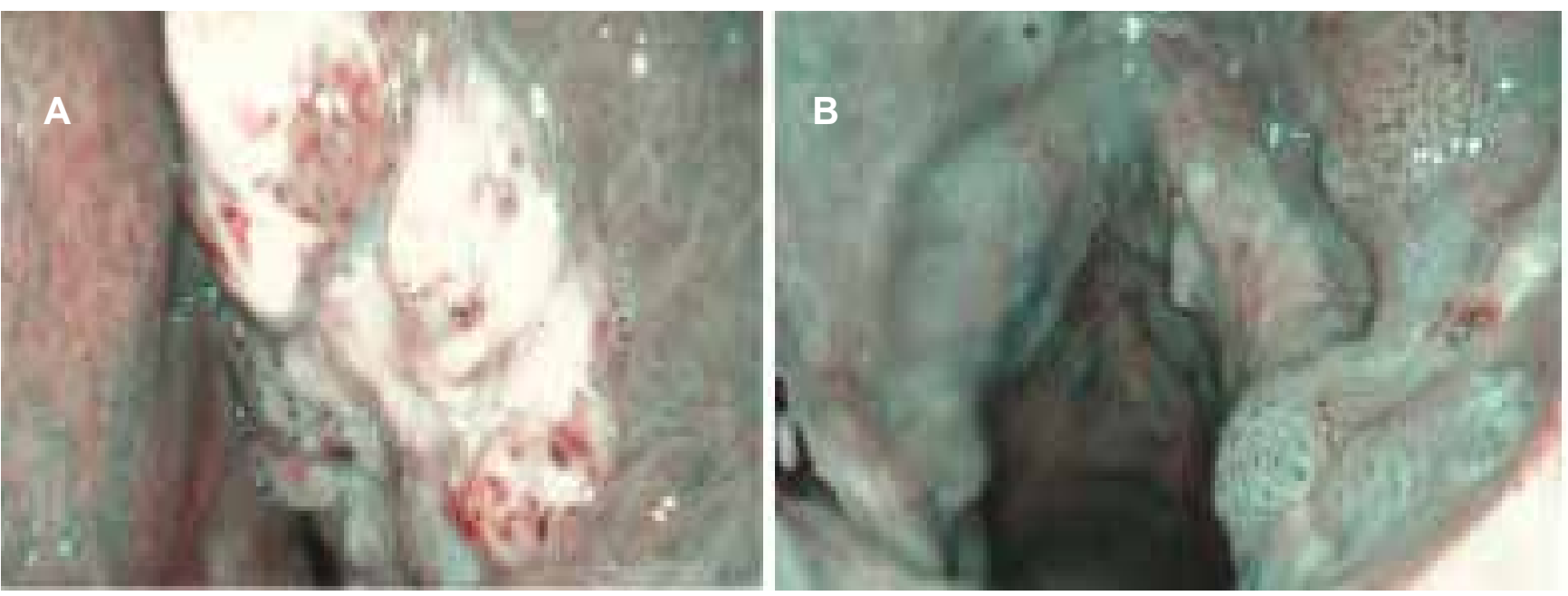

Figura 6. Cambios vasculares perpendiculares. A. Lesión de aspecto infiltrativo que compromete completamente la cuerda vocal derecha, con evidencia de bucles capilares intraepiteliales de ángulo estrecho. B. Lesiones de aspecto papilomatoso en el tercio posterior de la cuerda vocal izquierda y la banda ventricular izquierda, con evidencia de bucles capilares intraepiteliales de ángulo ancho. Imágenes cortesía del servicio de otorrinolaringología del Hospital de San José.

neoplásicos (patrón tipo $\mathrm{V}$ de $\mathrm{Ni}$ ) en estadios iniciales y facilita su localización para la realización de biopsias dirigidas $(6,17)$. Es importante tener en cuenta que la presencia de leucoplaquias oculta los capilares de la mucosa (efecto sombrilla), por lo que es necesario buscar alteraciones en los límites de la lesión con el epitelio normal (Figura 7).

\section{Corditis polipoide de Reinke}

Puede asociarse con una neoplasia intraepitelial escamosa debido a su relación causal con el tabaquismo.

\section{Laringitis hipertrófica}

En su forma de corditis en islotes, se aprecia como zonas engrosadas rojas y ovaladas diseminadas en el borde libre y superior de los pliegues vocales, lo que da lugar a una imagen irregular festoneada, cuyo diagnóstico diferencial es difícil de detectar a través de la exploración con luz blanca (7).

\section{Neoplasias malignas laringeas}

En los últimos años, la supervivencia de pacientes con cáncer de laringe no ha cambiado significativamente; por esta razón, es primordial realizar un diagnóstico temprano y la detectar las recidivas para mejorar la sobrevida a largo plazo (18-20). De forma global, el patrón tipo V (Clasificación de $\mathrm{Ni}$ ) corresponde a un carcinoma invasivo en el $84 \%$ de los casos, un carcinoma in situ en $6,8 \%$, una displasia grave en $4,5 \%$ y una displasia leve y moderada en $2,3 \%$ (8).

En el carcinoma infiltrante de laringe se ha visto una zona marginal carcinomatosa en el 30-50 \% de los casos; de estos, el $38-45 \%$ en la glotis y hasta el $73 \%$ en la supraglotis. Su tratamiento quirúrgico implica conocer el área específica del tumor, la zona marginal carcinomatosa y la zona metaplásica precancerosa. La NBI constituye una herramienta intraquirúrgica que facilita su identificación, así como de los límites oncológicos adecuados (21-23). 

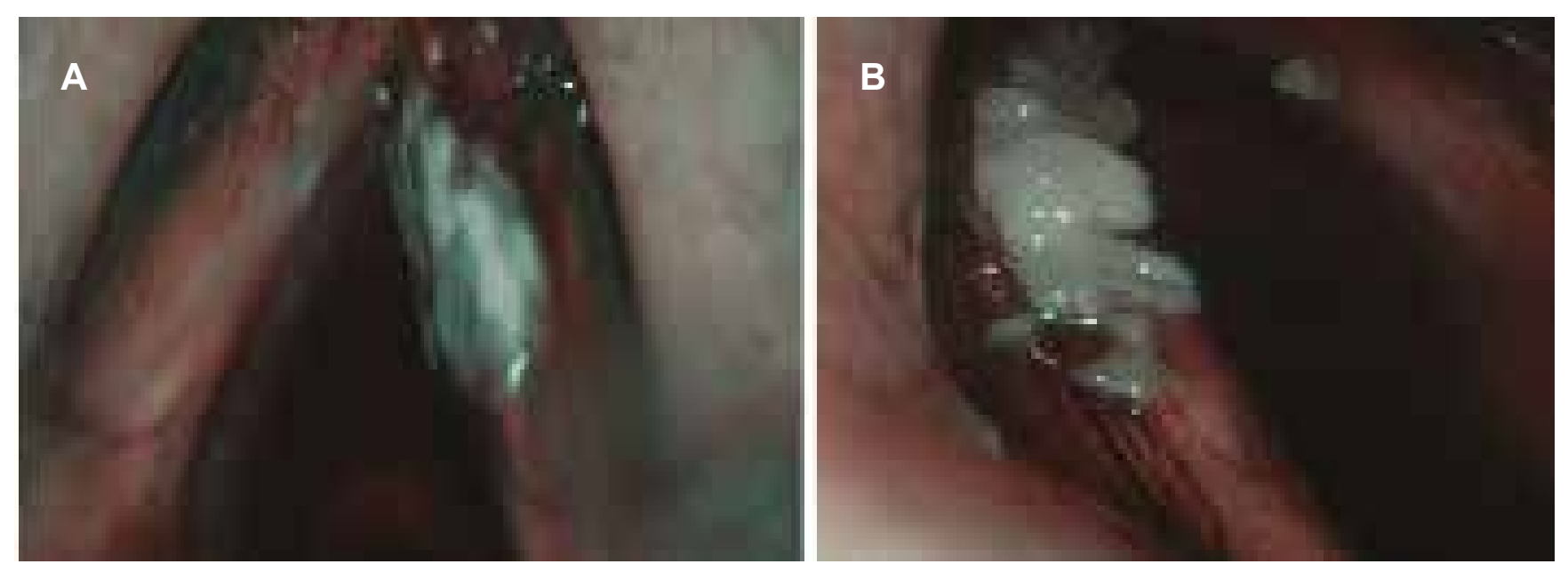

Figura 7. Efecto sombrilla. A. Leucoplaquia con luz blanca en el tercio medio de la cuerda vocal derecha con NBI, que evidencia vasos nutricios en la cuerda vocal izquierda y derecha con efecto sombrilla. B. Leucoplaquia con luz blanca en el tercio anterior y medio de la cuerda vocal izquierda con NBI, que evidencia vasos nutricios con efecto sombrilla. Imágenes cortesía del servicio de otorrinolaringología del Hospital San José.

Es importante recordar que la presencia de tejido necrótico, lesiones blanquecinas engrosadas o una distancia inadecuada pueden interferir con la identificación de los bucles capilares intraepiteliales. Para superar estas limitaciones, es importante acercar el endoscopio a las zonas que delimitan el carcinoma infiltrante, y dirigirlo a las áreas donde la mucosa sea más fina $(7,24)$.

Detección de carcinoma primario de células escamosas desconocido de cabeza y cuello

A pesar del desarrollo de técnicas de imagen sofisticadas que han mejorado significativamente la identificación de la lesión primaria, solo el 1,4-54 \% tienen un tumor detectable. Esto explicaría, en parte, por qué la supervivencia general de estos pacientes a 5 años oscila entre $35-50 \%(22,25,26)$.

Filauro y colaboradores encontraron que la sensibilidad, la especificidad y los valores predictivos positivos y negativos de la evaluación de la NBI fueron $91 \%, 95 \%, 91 \%$ y $95 \%$, respectivamente, mientras que la precisión diagnóstica fue del $90 \%(24,25,27)$. En este contexto, la NBI permitió la identificación de lesiones previamente no detectadas en el $34,5 \%$ de los pacientes. En este sentido, la NBI podría ser, en el consultorio, la prueba diagnóstica de primera línea en los pacientes con una biopsia por aspiración con aguja fina (BACAF) positiva, antes de someterlos a exámenes más costosos (25).

\section{Control clínico posterior al tratamiento con láser $\mathrm{CO}_{2}$}

La identificación de enfermedad recurrente o residual después de tratamiento con láser $\mathrm{CO} 2$ es difícil debido el proceso cicatricial normal. Además, se ha visto que el patrón de crecimiento de los carcinomas recurrentes es diferente, puesto que se presentan varios focos tumorales (multicén- tricos) localizados bajo una mucosa aparentemente poco alterada (28).

El seguimiento de pacientes con compromiso de la comisura anterior es difícil dada la posibilidad de cicatrices posoperatorias o granulomas, y porque pueden extenderse a zonas extralaríngeas. Por lo anterior, requieren de una vigilancia más estrecha para detectar estas recurrencias locales de forma precoz, y generalmente se realizan biopsias a las 6-8 semanas para su control. La NBI, aunque no sustituye la conducta anterior, es un avance en el control endoscópico, que evita múltiples biopsias y la posibilidad de deterioro de la voz (29).

\section{Control clínico posterior con radioterapia}

La radioterapia, en cualquiera de sus modalidades o en adyuvancia a la quimioterapia, produce cambios significativos en la mucosa y la submucosa, que limitan la valoración de las alteraciones que sugieren persistencia o recurrencia tumoral $(18,30-32)$.

En primer lugar, ocurre una toxicidad aguda dosis-dependiente, que produce un eritema que se transforma en mucosa con pseudomembranas. Posteriormente, aparece la toxicidad tardía como una combinación del daño del endotelio vascular, fibrosis cicatricial y atrofia muscular junto con la muerte celular (7).

Entre los cambios endoscópicos a mediano y largo plazo se encuentran edema difuso, áreas de hipervascularización, disminución de la secreción salival y zonas engrosadas por el aumento de fibrosis. Por lo anterior, se ha encontrado que las técnicas endoscópicas convencionales alcanzan porcentajes de diagnóstico tan bajos como $50 \%(29,33)$.

De esta manera, es necesario conocer las variaciones de las imágenes endoscópicas obtenidas mediante la NBI en estos pacientes, ya que esta mucosa, aun en ausencia de recurrencia o persistencia tumoral, no presenta el mismo 

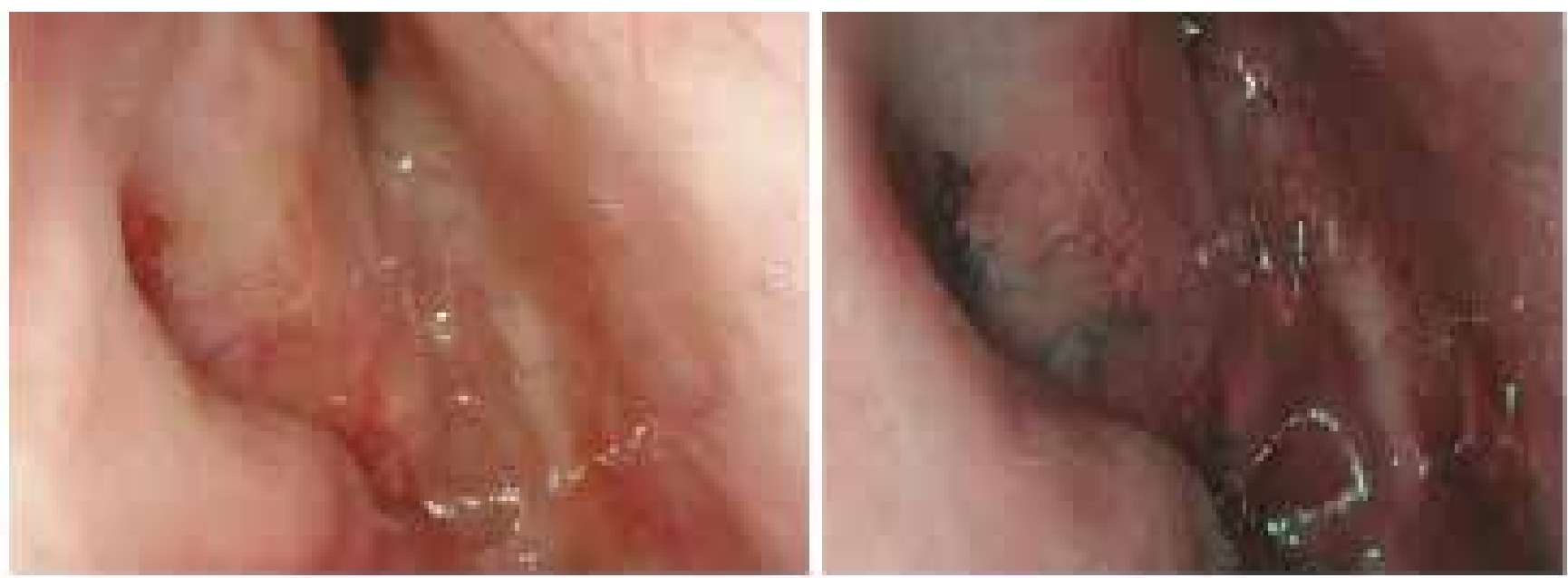

Figura 8. Imagen de NBI posradioterapia. A. Imagen con luz blanca. B. Imagen con NBI. Imágenes cortesía del servicio de otorrinolaringología del Hospital de San José.
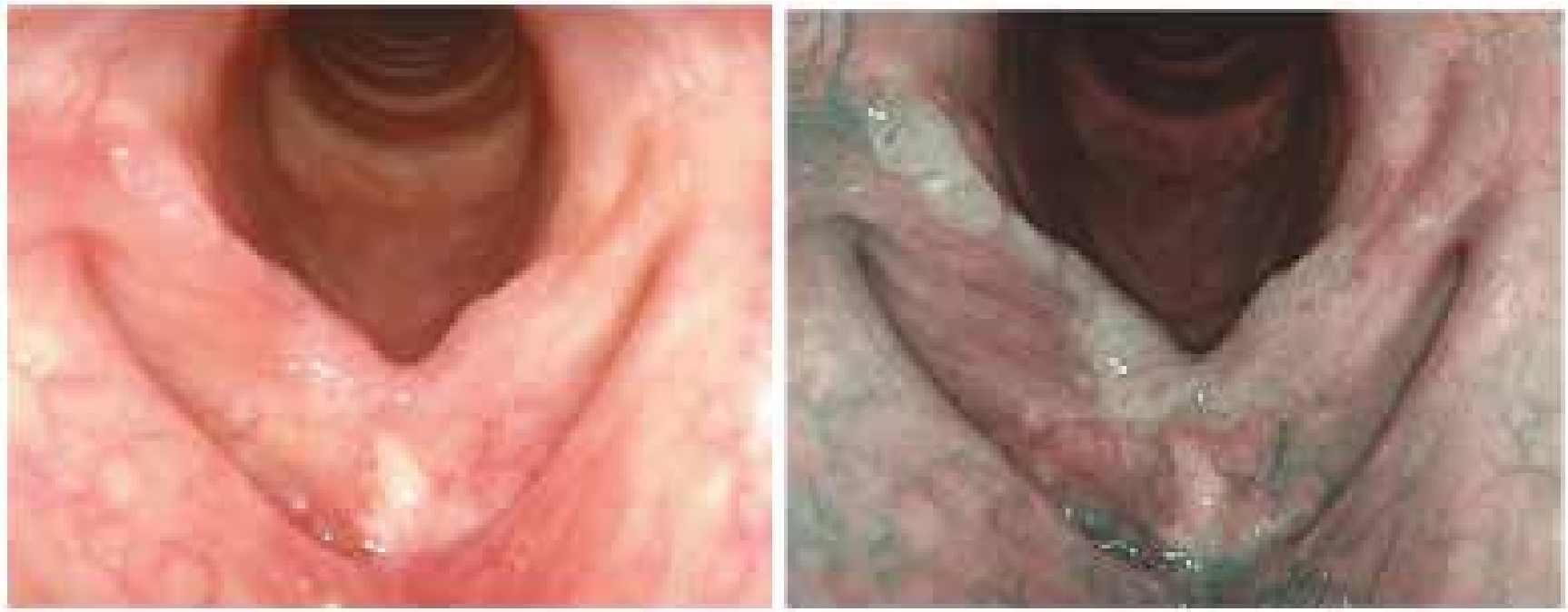

Figura 9. Papilomatosis respiratoria recurrente. A. Imagen con luz blanca. B. Imagen con NBI en la que se observan lesiones papilomatosas en el borde libre de los pliegues vocales. Imágenes cortesía del servicio de otorrinolaringología del Hospital de San José.

patrón vascular que la mucosa no tratada (7). En este caso, los neovasos adquieren formas arborescentes anárquicas con diámetros variados y zonas vacías de vascularización, que dan como resultado una imagen poco homogénea. En ocasiones, las zonas de edema se aprecian con cambios vasculares difusos de angiogénesis; y si no se distingue un límite claro entre el epitelio normal y el epitelio anormal, la imagen debe valorarse con precaución. En este sentido, hay que observar, además del patrón anormal sugestivo de neoplasia, la vascularización de las zonas limítrofes (Figura 8) (3, 34-36).

Piazza y colaboradores encontraron que la NBI mantiene su alta sensibilidad y especificidad incluso después de la radioterapia o la quimiorradiación; además, demostraron que, después de una curva de aprendizaje adecuada, los cambios posactínicos podrían distinguirse de manera confiable de las persistencias/recurrencias, lo que lleva a un número comparable (si no menor) de falsos positivos a los observados en los pacientes en seguimiento. De hecho, los patrones de IPCL de tipo II, III y IV de acuerdo con la clasificación de
Ni y colaboradores pueden asociarse con frecuencia a la inflamación posradioterapia, pero se distinguen fácilmente del patrón de tipo $\mathrm{V}$ (patognomónico de persistencia o recurrencia) $(37,38)$. Especialmente después de los protocolos de preservación de órganos de la laringe, esto se traduce en un número menor de biopsias injustificadas en la búsqueda de una enfermedad recurrente. Incluso después de la radiación o la quimiorradiación, la NBI mantiene valores extremadamente altos de sensibilidad, especificidad, valor predictivo positivo y negativo y precisión $(100 \%, 98 \%, 92 \%, 100 \%$ y $98 \%$, respectivamente) $(3,39,40)$.

\section{Papilomatosis respiratoria recurrente}

La papilomatosis respiratoria recurrente (PRR) es una enfermedad benigna causada por el virus del papiloma humano (VPH) 6 y $11(41,42)$. En la PRR, el estímulo de la infección epitelial en el tejido conjuntivo subyacente parece iniciar la neoangiogénesis y la transformación del propio tejido conec- 
tivo $(10,18,43)$.

To hay tratamiento curativo para la enfermedad. El objetivo del tratamiento es una extirpación quirúrgica total de las lesiones papilomatosas para reducir el número de recaídas. Por lo tanto, un buen método de visualización de los papilomas es crucial durante la cirugía $(43,44)$.

La NBI también puede ser útil en pacientes que sufren PRR, ya que, al aumentar el contraste entre el epitelio de la mucosa y los vasos submucosos, estas estructuras vasculares pueden reconocerse endoscópicamente como bucles capilares papilares intraepiteliales, con un ángulo ancho en una estructura verrugosa tridimensional (Figura 9) $(10,43,45)$.

\begin{tabular}{|c|c|}
\hline Presencia de sangre & \multirow{7}{*}{$\begin{array}{l}\text { Impiden la visualización de } \\
\text { los cambios de la mucosa y } \\
\text { la submucosa, aumento de la } \\
\text { mucosidad que altera la imagen } \\
\text { y no se logran visualizar los } \\
\text { patrones de angiogénesis }\end{array}$} \\
\hline Lesiones melánicas & \\
\hline Presencia de fibrina & \\
\hline Ulceraciones & \\
\hline Presencia de saliva & \\
\hline Lesiones hiperqueratósicas & \\
\hline Neoplasias de tejido linfoide & \\
\hline
\end{tabular}

\section{Conclusiones}

La NBI constituye una herramienta complementaria en la valoración de patologías malignas y benignas de la laringe, ya que identifica las lesiones superficiales de la mucosa que previamente se pueden pasar por alto con la endoscopia estándar. Su utilidad se ha demostrado en el seguimiento de pacientes con cáncer de laringe luego de un tratamiento con láser $\mathrm{CO} 2$, radioterapia y/o quimioterapia.

Se debe tener en cuenta la alta sensibilidad que tiene el uso de la NBI, que permite identificar de forma temprana lesiones neoplásicas y preneoplásicas con su extensión superficial en patología laríngea y mejorar la sobrevida de los pacientes con dicha patología.

Hemos estado utilizando de manera rutinaria la NBI en el servicio de otorrinolaringología del Hospital de San José para el diagnóstico de la patología laríngea, lo cual nos impulsó a realizar esta revisión. En ese contexto, se necesitan más estudios en el país que exploren este tipo de imagen endoscópica y su uso en laringología.

\section{REFERENCIAS}

1. Stachler RJ, Francis DO, Schwartz SR, Damask CC, Digoy GP, Krouse HJ, et al. Clinical Practice Guideline: Hoarseness (Dysphonia)(Update). OtolaryngolNeckSurg.2018;158(3):409426. https//doi.org/10.1177/0194599817751031

2. Martins RHG, Hidalgo Ribeiro CB, Fernandes de Mello BMZ, Branco A, Tavares ELM. Dysphonia in Children. J Voice. 2012;26(5):674.e17-674.e6.74E20. https//doi.org/10.1016/j. jvoice.2012.03.004
3. Choi SS, Zalzal GH. Voice disorders in children. En: Cummings Jtolaryngology, Head \& Neck Surgery. 6.a ed. Filadelfia: Elsevier; 2015. p. 3133-41.

4. Gray SD, Smith ME, Schneider H. VOICE DISORDERS IN CHILDREN. Pediatr Otolaryngol. 1996;43(6):1357-1384. https//doi.org/10.1016/s0031-3955(05)70523-x

5. Levitsky SE. Hoarseness. En: Primary pediatric care. 4.a ed. St. Louis: Mosby; 2001. p. 1156.

6. Yang J, Xu W. Characteristics of Functional Dysphonia in Children. J Voice. 2020;34(1):156.e1-156.e4. https//doi. org/10.1016/j.jvoice.2018.07.027

7. Angelillo N, Di Costanzo B, Angelillo M, Costa G, Barillari MR, Barillari U. Epidemiological study on vocal disorders in paediatric age. J Prev Med Hyg. 2008;49(1):1-5.

8. Connelly A, Clement WA, Kubba H. Management of dysphonia in children. J Laryngol Otol. 2009;123(6):642-7. https//doi. org/10.1017/S0022215109004599

9. Toohill RJ. The Psychosomatic Aspects of Children With Vocal Nodules. Arch Otolaryngol. 1975 Oct;101:5. https//doi. org/10.1001/archotol.1975.00780390005002

10. Wilson DK. Children with Vocal Nodules. J Speech Hear Disord. 1961;26(1):19-26.

11. Batza EM. Vocal Cord Nodules in Children: Pathogenesis, Clinical Manifestations, Therapy. Clin Pediatr (Phila). 1970;9(1):14-6. https//doi.org/10.1177/000992287000900105

12. Kay NJ. Vocal nodules in children - aetiology and management. J Laryngol Otol. 1982;96:731-6. https//doi.org/10.1017/ s0022215100093051

13. Martins RHG, do Amaral HA, Tavares ELM, Martins MG, Gonçalves TM, Dias NH. Voice Disorders: Etiology and Diagnosis. J Voice. 2016;30(6):761.e1-761.e9. https//doi. org/10.1016/j.jvoice.2015.09.017

14. Nardone HC, Recko T, Huang L, Nuss RC. A Retrospective Review of the Progression of Pediatric Vocal Fold Nodules. JAMA Otolaryngol Neck Surg. 2014;140(3):233. https//doi. org/10.1001/jamaoto.2013.6378

15. Nunes RB1, Behlau M, Nunes MB, Paulino JG. Clinical diagnosis and histological analysis of vocal nodules and polyps. Braz J Otorhinolaryngol. 2013;79:434-40. https//doi. org/10.1001/jamaoto.2013.6378

16. Teachey JC, Kahane JC, Beckford NS. Vocal mechanics in untrained professional singers. J Voice. 1991;5(1):51-6.

17. Qua CS, Wong CH, Gopala K, Goh KL. Gastro-oesophageal reflux disease in chronic laryngitis: prevalence and response to acid-suppressive therapy. Aliment Pharmacol Ther. 2007;25(3):287-295. https//doi.org/10.1111/j.13652036.2006.03185.x

18. Kahrilas PJ, Shaheen NJ, Vaezi MF. American Gastroenterological Association Medical Position Statement on the Management of Gastroesophageal Reflux Disease. Gastroenterology. 2008;135(4):1383-1391.e5. https//doi. org/10.1053/j.gastro.2008.08.045

19. Hopkins C, Yousaf U, Pedersen M. Acid reflux treatment for hoarseness. Cochrane Database Syst Rev. 2006;(1):CD005054. https//doi.org/10.1002/14651858.CD005054.pub2 